\title{
Remote Laboratories: AIP-Primeca RAO Platform
}

\author{
P. COQUARD, M. GUILLEMOT, D. NOTERMAN \\ AIP-Primeca RAO \\ Bat. G. Jacquard, 10 rue des sports \\ 69621 Villeurbanne Cedex, France \\ coquard@aiprao.insa-lyon.fr
}

\author{
A. LELEVE, H. BENMOHAMED \\ INSA Lyon \\ Bat. L. de Vinci, 21 avenue Jean Capelle \\ 69621 Villeurbanne Cedex, France \\ arnaud.leleve@insa-lyon.fr
}

\begin{abstract}
During last decade, Internet and related web technologies development enabled the arising of e-learning services and made distant learning a reality. As traditional face to face classroom became virtual classroom through Internet, traditional laboratories found their equivalent in eLaboratories. These e-Labs enable learners to train on remote real or virtual systems. They represent essential components in e-learning environments, especially in scientific and technical disciplines.

In this context, AIP-Primeca RAO is a pool of resources and competencies for industrial topics for many universities in Rhone. Due to the constraints of using heavy and shared resources, AIP is setting up new laboratories related to automation as both local and distant resources.

After recalling the global context of e-laboratories, this paper describes this platform. It evokes first returns of use and it depicts evolutions to come.
\end{abstract}

\section{Introduction}

For a few years, numerous educational institutions have incorporated tools based on Information and Communication Sciences and Technologies into their educative systems. These new tools contribute to a continuous improvement of educational practices. So, e-learning solutions, which were at first essentially based on abstract education (online courses, virtual classrooms, e-projects, role-playing, ...), gradually opened themselves to real hands-on activities through Electronic Laboratories (E-Labs) [12]. This trend answers to a recognized need for such activities and enables real experimentation in scientific and technical disciplines.

In this context, AIP-Primeca RAO (Atelier Interetablissements de Productique Rhone-Alpes Ouest) has been a pool of resources and competencies setting industrial equipment at the common disposal of many high schools from Rhone as a support for thorough trainings in industrial engineering since 1987. The 8 partner universities are also its main users in Lyon (INSA, Ecole Centrale, University Claude Bernard Lyon 1, ECAM, IUT Lumiere of University Lyon 2) and in Saint-Etienne (ENI, Ecole des Mines, University). The resources set at users disposal are mainly composed of :

- industrial equipment (such as CNC machine-tool, conveyors, assembly automation systems, robots, ...);

- computer systems supporting industrial software (CAD-CAM, simulation, CAPP, shop control, ...);

- communication and cooperation systems (LAN, WAN, video-communication, Lotus Notes, ...).

AIP has been studying solutions to promote laboratories to ELabs since 2000 [13, 20]. Since 2006, a growing need for laboratories about automation technologies (Programmable Logic Controller (PLC), Human Machine Interface (HMI) programming, Industrial Local Area Network (ILAN) setting, motion control, ...) from different users at INSA Lyon and from Schneider Electric has led to open real E-Labs (ARI project). The reasons and the main concepts of this project are described in section 3.1 and 3.2. To sum up, it consists of providing both local and distant access to sets of apparatuses (PLC+HMI+ILAN+Motion control). The means to teleoperate these apparatuses are currently available online from the AIP portal. The corresponding architecture is detailed in section 3.3, first use returns are commented out in 3.4.

In a near future, this platform will be completed with elearning software developed by LIESP e-learning team (exICTT), to provide fully functional local and remote laboratories. This evolution is given in section 4 .

\section{Electronic laboratories}

Electronic laboratories (E-Labs), are divided in two categories: Remote Laboratories (RLabs) $[4,15]$ and Virtual 
Laboratories (V-Labs) [10, 16]. R-Labs (sometimes called web-based control), offer remote access to real laboratory equipment and instruments in real time. V-Labs are based on simulations of real systems or phenomena. These simulations can be run directly on client host such as with Easy Java Simulation [11]. R-Lab and V-Lab Human Machine Interfaces are often supplied by commercial software such as LabView [2] or Matlab+Simulink [8], or by specifically designed software such as for [1]. Notice that some R-Labs may include Virtual Reality techniques [17] which lead this kind of laboratories to be part of a third category of laboratories: Hybrid Laboratories (H-Labs), combining advantages of both previous categories. Differences, advantages and drawbacks of R-Labs versus V-Labs have already been discussed in litterature and more precisely in [7].

Many R-Labs deal with electronics [18], automatic control [3] and robotics [14] but fewer with discrete state systems. We can however cite $[4,6,17,19]$ who proposed solutions for these kinds of systems.

Current generation of research about E-Labs is no more based uniquely on teleoperation of devices but it also takes care of actors (authors, tutors and learners) educational goals, from scenario authoring to experimental result reporting and evaluation, as for [9] and [18]. In this context, some works on linking traditional Learning Management Systems (LMS) with E-Lab Management Systems (ELaMS) are on way at LIESP and DIOM laboratories [12].

\section{ARI project}

\subsection{Initials needs}

AIP has already studied collaborative means to help people to work together and at distance, more particularly on production systems. In this context, we launched, in 2006, the ARI project in collaboration with 3 departments of INSA (Electrical Engineering, Industrial Engineering and Mechanical Engineering and Design), long life education centre INSACAST and Schneider Electric. This project consists in building a technological platform featuring modern controllers and industrial networks in the aim to illustrate the concept of the Transparent Factory: Internetbased technologies that provides seamless communication between plant-floor and business systems and improves collaborative management.

This shared and centralized platform has been designed to be accessible at distance by every member educative institution and other socio-economic actors (partners and clients) in the frame of initial and long-life learning. This realization is set up on the experience acquired during previous projects, one of them with nomadic students working with CNED $^{1}$ institute.

\footnotetext{
${ }^{1}$ Distance Learning Institute; see http : / www . cned.fr/en/
}

Needs from different partners are summed up here:

- for initial learning (INSA, ECAM, ...):

- development of student autonomy for self education;

- experience of industrial software engineering (functional analysis, design, programming, control);

- for long-life learning

(alternately or continuous education: $\mathrm{CNAM}^{2}$, $\mathrm{ITII}^{3}$ ):

- supply, to students in alternance in their enterprise, of access to software and hardware resources, outside office schedules (night and week-end);

- training cost optimization:

* decrease of time of employees away from their enterprise;

* validation of prerequisites and verification of adequation between trainee competences and training program before training periods;

* access to the apparatus after training periods to increase trainee acquisition of knowledge or enable a preparation for an exam or a certification;

- for partners:

- supply of distant access to shared equipment;

- development of a competent team federating the actors of education in the different institutes;

- providing of a technological showcase to train experts and managers about concepts of digital enterprise;

- common needs:

- decrease of time spent in transports for far users;

- raise of apparatus busy rate.

\subsection{Concept}

This platform enables control of automation devices such as motors, variable speed drives, pneumatic actuators, ... by Programmable Logic Controllers (PLC) through Industrial Local Area Networks. It is built according to a modular architecture. Every module illustrates a modern

\footnotetext{
${ }^{2}$ Lifelong Learning Inst.: http: //www. cnam.fr/42394825/0/ fiche_-_pagelibre/

${ }^{3}$ Lifelong Learning Inst: http://www.uimm.fr/fr/reseau/ contenu_ITII.html
} 
ILAN (Modbus/RTU, Modbus/TCP, CanOpen, Profibus, Ethernet TCP/IP and AS-i). They are all built according to the same structure: a PLC : fitted with an Ethernet coupler featuring a web server; connected through an ILAN to some distributed sensors and actuators. Every module (depicted on figure 1) can be extended with complementary equipment according to specific needs (other sensors, actuators, position encoders, ...).

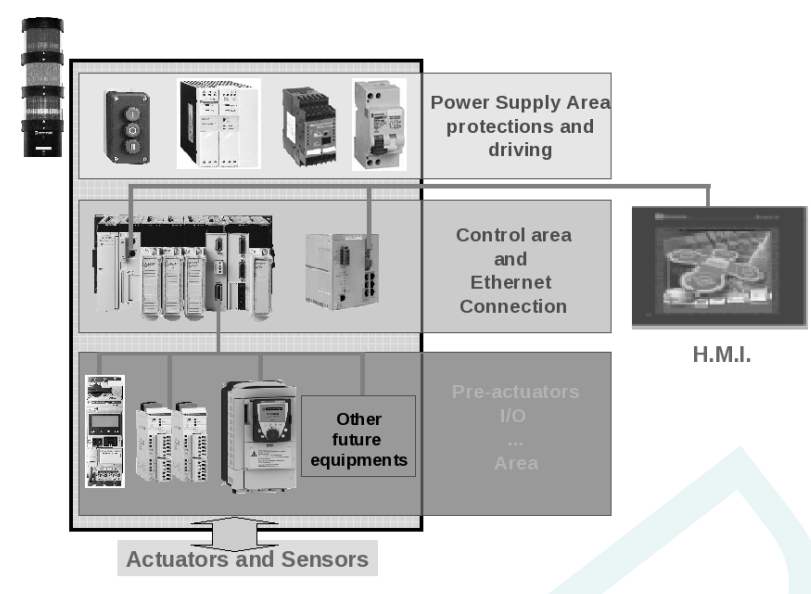

Figure 1. Module generic architecture

Thanks to Ethernet couplers, modules can be connected together in order to create more complex architectures.

This platform is available both locally and at distance. Distant users gain access through AIP web portall. Specific web services have been developed to manage and schedule access to these modules. Moreover, some modules are physically present at AIP and some others are located in Electrical Department of INSA (on the same campus). Assembly will obviously be available only between neighbours but single module transparent access will be provided on web portal as if modules were all together.

Global architecture is detailed in next section. Currently, 3 types of laboratories are available:

- 2 surface treatment modules with As-I;

- 1 Profibus module with a motion controller and digital I/O which can be driven through exploitation screens;

- 1 Ethernet + Modbus with a networked motor started and a motor speed controller. The driving is performed through applets hosted on the Ethernet coupler on the PLC.

\subsection{Architecture}

Access to the ARI platform is provided through AIP web portal (see figure 4) according user profiles (visitor, stu-

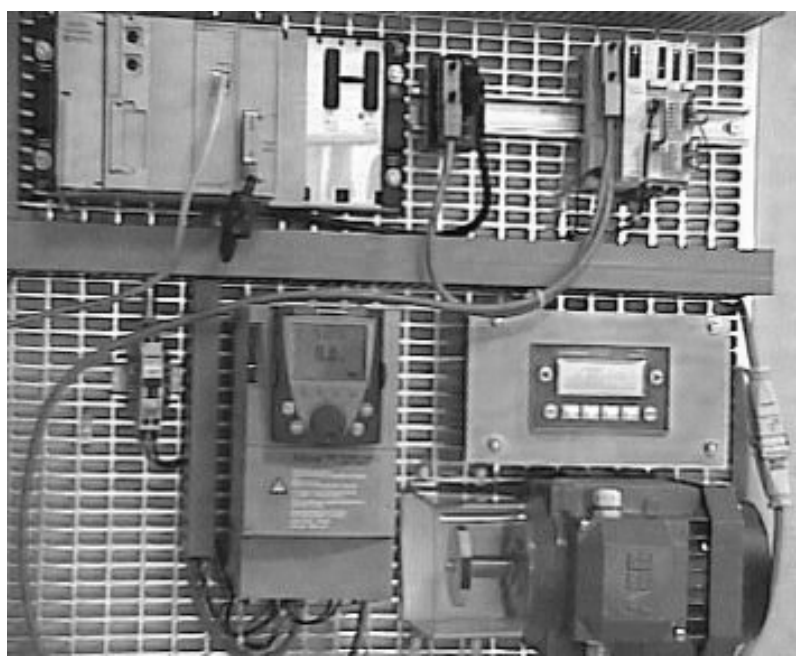

Figure 2. Profibus module

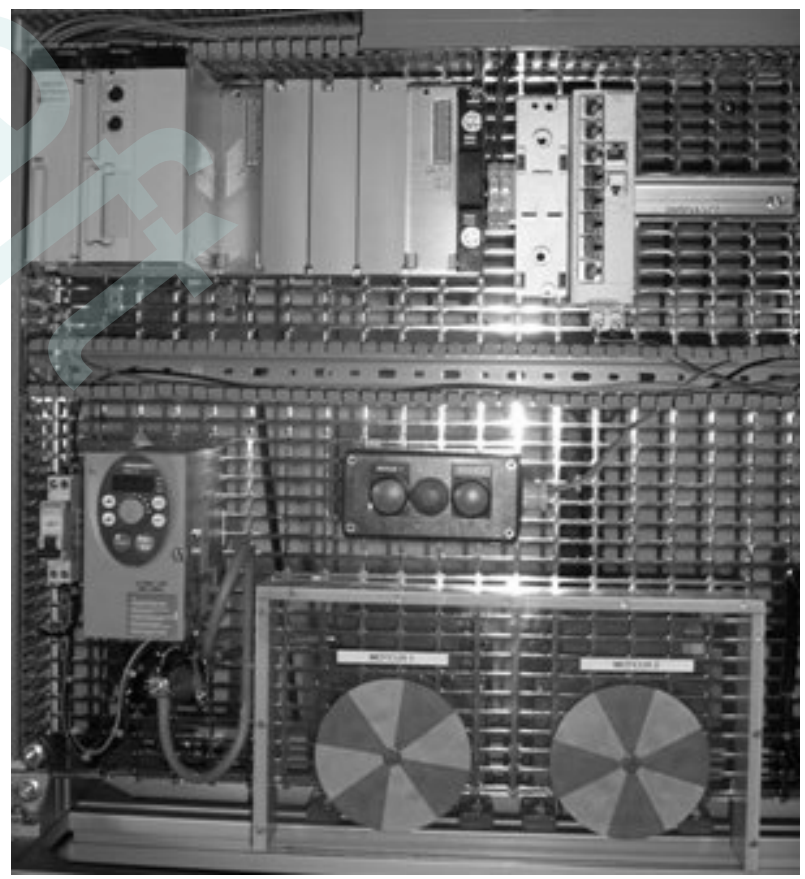

Figure 3. Modbus module 
dent, tutor, administrator). Users are recorded in AIP LDAP repository. Portal uses TomCat application server.

Visitors can give their name and email in order to have access to demonstrations of modules whenever they are not previously booked. In fact, it consists of having access to a limited amount of time (60min max) to every functionality of the platform.

Once connected into the intranet, tutors can book some modules for their laboratories. They are given the list of available resources according to a time period, the necessary documentation to help them in using proposed software. They can add additional documentation for their students (demonstration programs, complementary documentation, to-do lists, ...). They are given a schedule summing up their own bookings.

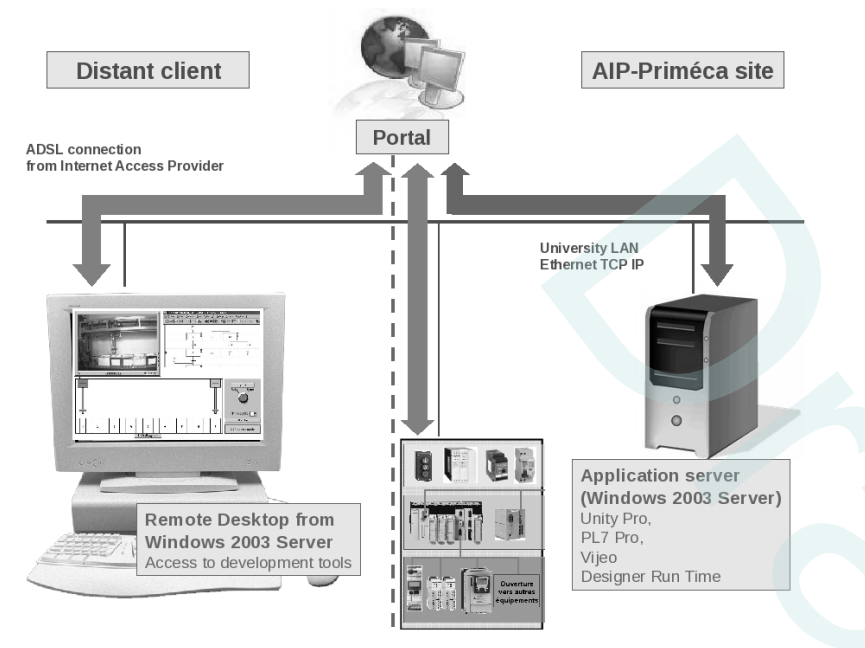

Figure 4. Platform architecture

Students have to provide a password given by their tutor to gain access to a specifically booked module. Once connected, they are given a single screen shared into webcam view, resources (documents, links, ...), the programming software and a Human Machine Interface (HMI) permitting to remotely test their programs (see figure 5). Their access is limited to the booked period. They produce documents (programs, reports, ...) during their session. Theses documents are stored on server part. They can also download them on their own desktop.

PLC programming is performed with the help of Schneider Electric Unity Pro development software. It is compliant with IEC 61131-3 standard and enables component based engineering. This software is installed on Terminal Server Edition (TSE) on a Windows 2003 Server, which permits distant users to use it remotely without having to install it on their own PC. It features an operative part simulator in order to preventively test one's program before upload it on the PLC through Ethernet LAN.
Once a program uploaded, real time handling of each module is performed through a HMI such as panel for local use and a virtual version of the same HMI for distant users. These HMI are designed with the help of supervision software such as Vijeo Designer and Citect Scada.

The whole architecture is based on J2EE environment. Web services enable a modular architecture able to be extended without having to redesign the whole web site from scratch. Active $\mathrm{X}$ components are used to gain access to the Terminal Server. This constrains users to use Microsoft Internet Explorer.

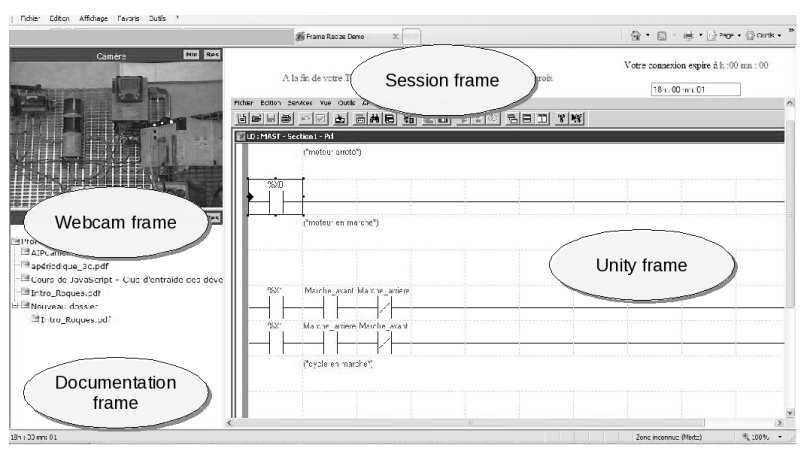

\section{Figure 5. Screenshot of student interface to module}

\subsection{Usage returns}

2006-2007 academic year enabled to begin the deployment of prototypes for local and distant use with As-I, Modbus and Profibus ILANs. 36 students from Licence at University Lyon 1 (same campus) remotely used this platform. This first experimentation enabled us to validate first laboratory scenarios and led to make adjustments about distant means. For example, for laboratories open out of business hours, it is necessary to drive the lighting to be able to use efficiently the web cam... Tests at greater distances, from Nantes $(650 \mathrm{~km})$ and Seville in Spain $(1630 \mathrm{~km})$ showed us that such distances do not constrain the ergonomy in nomadic usages.

\section{Evolution}

From technical point of view, we remarked that Active $\mathrm{X}$ components could be a constraint on student and tutor accessibility. A new version based on Web 2.0 technologies is under development to enhance accessibility.

Some new modules will be developed to complete this set so that an entire class of students will be able to perform simultaneously their laboratory work. Also, as modules are 
currently available only as single devices. The ability to book and use linked modules will be available soon.

At now, means to teleoperate modules are available but no learning help is provided to users (tutors and learners).

We are going to implement works on generic E-Lab scenarios from [5] to provide an E-Lab compliant laboratory Learning Management System.

The aim of this evolution is to provide a way for authors and tutors to edit generic E-Lab scenarios without requiring to be computer science specialists.

The key concept is a middleware called ElaMS (Electronic Laboratory Management System) which plays the role of driver manager according to printer analogy. It permits E-Lab scenario sharing as for any standard pedagogical content. In this context, an author writes a standard generic scenario which is linked to a class (a template) of apparatuses (PLC + motor, oven, robot, ...) which, in its turn, is represented by a set of available standard online functionalities. This scenario can be run on a standard (in our case, IMS-LD compliant) LMS and its activities appeal to specific functionalities of a fictive apparatus. When a tutor wants to execute it on its platform, he just has to run an automated scenario adaptation script which transforms this generic scenario into an apparatus specific scenario by changing its generic functionality calls into real function calls towards his apparatus.

This life cycle is depicted in figure 6. It consists of four main steps:

- installation (by a platform manager) of a new apparatus on the ELaMS platform; this apparatus must belong to a known apparatus class;

- creation (by authors) of generic pedagogic scenarios on an authoring tool; each scenario is linked to an apparatus class;

- adaptation (by tutors) of a generic pedagogic scenario to a specific apparatus and ;

- use (by tutors and learners) of these scenarios on this specific apparatus.

By using an LMS and scenarios, learners will be then more finely guided during their lab work and tutors will be able to follow at distance how far learners have made headway. LMS can be also used to help in assessment.

\section{Conclusion}

This paper outlines ARI project at AIP-Primeca RAO where a platform including modular apparatuses featuring PLC and Industrial Networks is being built. These apparatuses are to be used in situ and at distance. The global architecture from client to PLC was depicted and evolutions to

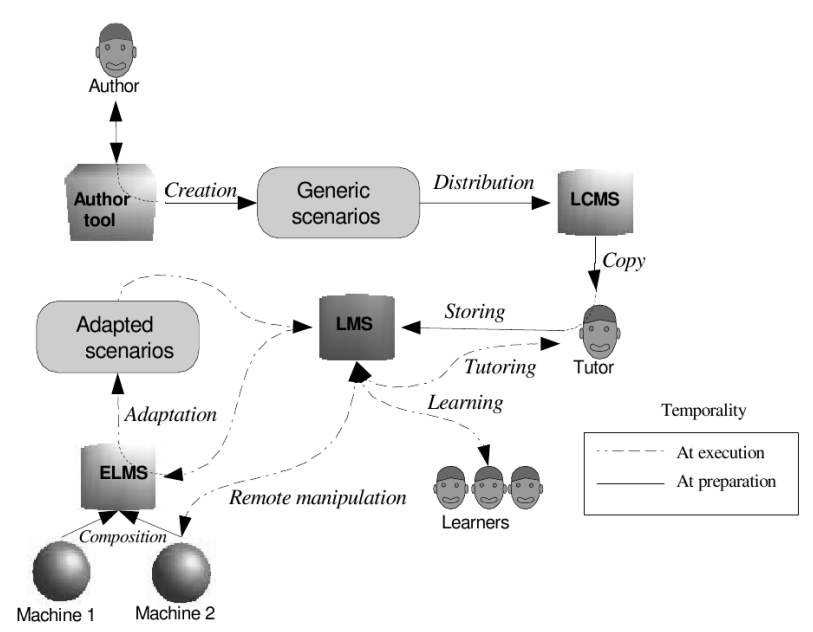

\section{Figure 6. Typical life cycle for E-Lab scenarios}

come were exposed. This architecture covers teleoperation layer. Next developments will lead to provide e-learning layer on top of previous one to accompany learners and tutors in their practical activities.

\section{References}

[1] G. Andria, A. Baccigalupi, M. Borsic, P. Carbone, P. Daponte, C. De Capua, A. Ferrero, D. Grimaldi, A. Liccardo, N. Locci, A. M. L. Lanzolla, D. Macii, C. Muscas, L. Peretto, D. Petri, S. Rapuano, M. Riccio, S. Salicone, and F. Stefani. Remote didactic laboratory "g. savastano": the italian experience for the e-learning at the technical universities in the field of the electrical and electronic measurements, overview on didactic experiments. In Instrumentation and Measurement Technology Conference, 2006. IMTC 2006. Proceedings of the IEEE, pages 1537-1542, 2006.

[2] A. Baccigalupi, C. De Capua, and A. Liccardo. Overview on development of remote teaching laboratories: from labview to web services. In Instrumentation and Measurement Technology Conference, 2006. IMTC 2006. Proceedings of the IEEE, pages 992-997, 2006.

[3] A. Bauchspiess, B. Guimaraes, and H. Gosmann. Remote experimentation on a three coupled water reservoirs. In Industrial Electronics, 2003. ISIE '03. 2003 IEEE International Symposium on, volume 1, pages 572-577 vol. 1, 2003.

[4] O. Bellmunt, D. Miracle, S. Arellano, A. Sumper, and A. Andreu. A distance plc programming course employing a remote laboratory based on a flexible manufacturing cell. Education, IEEE Transactions on, 49(2):278-284, 2006.

[5] H. Benmohamed, A. Leleve, and P. Prevot. Generic framework for remote laboratory integration. In Information Technology Based Higher Education and Training, 2005. ITHET 2005. 6th International Conference on, pages T2B/11T2B/16, 2005. 
[6] D. Buhler, W. Kuchlin, G. Grubler, and G. Nusser. The virtual automation lab-web based teaching of automation engineering concepts. In Engineering of Computer Based Systems, 2000. (ECBS 2000) Proceedings. Seventh IEEE International Conference and Workshopon the, pages 156-164, 2000.

[7] J. Corter, J. Nickerson, S. Esche, and C. Chassapis. Remote versus hands-on labs: a comparative study. In Frontiers in Education, 2004. FIE 2004. 34th Annual, pages F1G-17-21 Vol. 2, 2004.

[8] M. de Magistris. A matlab-based virtual laboratory for teaching introductory quasi-stationary electromagnetics. Education,IEEE Transactions on, 48(1):8188, 2005.

[9] D. Gillet, A. V. N. Ngoc, and Y. Rekik. Collaborative web-based experimentation in flexible engineering education. Education, IEEE Transactions on, 48(4):696-704, 2005.

[10] S. K. Goudos, T. Samaras, and J. N. Sahalos. Web based laboratory in electromagnetic compatibility using a java applet. Computer Applications in Engineering Education, 14(4):269280, 2006.

[11] A. Grau and Y. Bolea. Virtual laboratory for modeling systems: A sustainable approach. In 32nd Annual Conference on IEEE Industrial Electronics (IECON 2006), page 478483, Paris, France, 2006. IEEE. November 7-10, 2006.

[12] C. Gravier, J. Fayolle, G. Noyel, A. Leleve, and H. Benmohamed. Distance learning: Closing the gap between remote labs and learning management systems. In E-Learning in Industrial Electronics, 2006 1ST IEEE International Conference on, pages 130-134, 2006.

[13] M. Guillemot, A. Lelev, D. Noterman, P. Prevot, and C. Subai. Towards remote laboratory platforms with dynamic scenarios. In 7th World Multiconference on Systematics, Cybernetics and Informatics (SCI 2003), jul 2003.

[14] E. Guimaraes, A. Maffeis, J. Pereira, B. Russo, E. Cardozo, M. Bergerman, and M. Magalhaes. Real: a virtual laboratory for mobile robot experiments. Education,IEEE Transactions on, 46(1):3742, 2003.

[15] T. Kikuchi, S. Fukuda, A. Fukuzaki, K. Nagaoka, K. Tanaka, T. Kenjo, and D. Harris. Dvts-based remote laboratory across the pacific over the gigabit network. Education, IEEE Transactions on, 47(1):26-32, 2004.

[16] F. Kuester and T. C. Hutchinson. A virtualized laboratory for earthquake engineering education. Computer Applications in Engineering Education, 15(1):1529, 2007.

[17] A. Lelev, C. Meyer, and P. Prvot. Tl-tp : premiers pas vers une modlisation. In Actes du 3me colloque international sur les Technologies de l'Information et de la Connaissance dans l'Enseignement suprieur et l'industrie (TICE 2002), Lyon, France, November 2002.

[18] S. Rapuano and F. Zoino. A learning management system including laboratory experiments on measurement instrumentation. Instrumentation and Measurement, IEEE Transactions on, 55(5):1757-1766, 2006.

[19] C. Saygin and F. Kahraman. A web-based programmable logic controller laboratory for manufacturing engineering education. The International Journal of Advanced Manufacturing Technology, 24(7):590-598, Oct. 2004.
[20] K. Szafnicki, M. Guillemot, G. Louail, and D. Noterman. Remote control of manufacturing systems - a collaborative course between engineering schools. In IFAC Symposium on Advances in Control Education (ACE 2000), Gold Coast, Australia, December 2000. 